\title{
Facial Cell Assisted Lipograft Comparing Mechanical and Enzymatic Preparation of the Stromal Vascular Fraction: A Comparative Study
}

\author{
ALY HUSSEIN SABER ABULHASSAN, M.B.B.Ch.1; HAMED MOHAMMED KADRY, M.D. ${ }^{2}$; \\ LOBNA YOUSEF GHANEM, M.D.3; MAAMOUN ISMAIL MAAMOUN, M.D.4; \\ AHMED ADEL NOURELDIN, M.D. $\mathbf{4}^{4}$ and HUSSEIN SABER ABULHASSAN, M.D. $\mathbf{5}$ \\ The Department of Plastic \& Reconstructive Surgery, Faculties of Medicine, Alexandrial,5 \& Cairo 2,4 Universities and \\ The Department of Clinical Pathology, Theodor Bilharz Institute 3
}

\begin{abstract}
Background: For more than a century, clinicians have attempted to use fat for the treatment of tissue deficiencies and contour abnormalities with variable outcome and fate of the transferred fat. Autologous fat transfer for facial contouring has become popular in plastic surgery only in the past 20 years. In fact, surgeons have understood that ageing of the face is not attributable to gravity-induced ptosis alone, but is also a consequence of volume loss caused by tissue atrophy. Thus, volume replacement using fat transfer is the aim of the hereby presented treatment.
\end{abstract}

Aim of the Study: To evaluate the effect of two types of isolation of lipograft in the face and their persistence and the changes they will produce.

Material and Methods: This study was conducted on 50 patients complaining of facial ageing and facial scars whether post traumatic or post burn from the period between January 2013 and December 2014. The sample population was divided into two groups:

- Group A: Underwent fat processing using the mechanical mesh/gauze to separate the SVF (39 patients) in order to obtain micro and nanofat grafts.

- Group B: Underwent fat processing using the enzymatic /collagenase technique to separate the SVF (11 patients).

Key Words: Lipograft - Stromal vascular fraction.

\section{INTRODUCTION}

The techniques of fat processing both the mechanical and enzymatic techniques have demonstrated valuable clinical outcomes in all indications. Although the enzymatic technique demonstrated valuable results in concentrating the ADSC's with less need for secondary retouches.

Autologous fat transplantation has been used to correct subcutaneous lipoatrophy resulting from acne, trauma, lipodystrophy, hemifacial atrophy, cutaneous lupus erythematosus, and scleroderma and defects resulting from accidents, infections, or surgery.
While autologous fat transfer for facial contouring has become popular in plastic surgery only in the past 20 years. In fact, surgeons have understood that ageing of the face is not attributable to gravityinduced ptosis alone, but is also a consequence of volume loss caused by tissue atrophy. Thus, volume replacement is the aim of the hereby presented treatment.

The use of adipose tissue for this purpose has become the preferred option, as it is readily available, inexpensive, host-compatible, and can be harvested easily and repeatedly when needed, without fear of allergies or foreign body reactions.

Despite clinical optimism associated with autologous fat transfer, uncertainty remains among practitioners regarding the viability of transplanted fat. There is no set way of processing fat to ensure the graft's viability and optimal take, although various preparation techniques have been suggested for improving the long-term survival of fat grafts.

The commonly used method of ADSC is the enzymatic digestion of the lipoaspirate. Although the major difference from the enzymatic technique, the ease of the mechanical separation of the ADSC which made the technique more popular.

\section{Adipose-derived stem cells:}

A population of multipotent cells exists within the stroma of human adipose tissue that closely resembles bone marrow derived mesenchymal stem cells and has demonstrated the ability to differentiate along adipogenic, chondrogenic, and osteogenic lineages. Adipose-derived stem cells (ASCs) are abundant and easily accessible, and they have been heavily promoted for their potential role in the regenerative medicine [1]. 
It has been shown that the number of ASCs obtained via liposuction is about half the number found in excised whole fat. This is believed to be due to ASC concentration around areas of blood vessels, which are mostly left behind during liposuction harvest. The relative deficiency in ASCs after liposuction could be a major factor when it comes to unpredictable graft take. Yoshimura et al., attempted to overcome the unpredictability of lipoassisted fat transfer by augmenting the infiltrate with a greater number of ASCs [2]. In their technique, autologous ASCs are isolated from half of the aspirated fat and combined with the other half of the harvested fat to "supercharge" the injected graft with ASCs. This enriches the ASC content of grafted fat and may help promote angiogenesis in the recipient tissues. Their preliminary results with cell-assisted lipotransfer have been encouraging, but long-term data have not been published to allow any definitive conclusions [2].

Stem cells and operative time: Novel techniques in isolation.

\section{Enzymatic separation:}

The commonly used method of isolating MSC from adipose tissue is enzymatic digestion that consists of at least four main steps: Digestion, washing, centrifugation and red blood cell lysis. Adipose tissue from lipoaspirate samples is incubated with collagenase for up to 1 hour. Then, the digests are washed, and centrifuged to separate the floating adipocytes from the pelleted stromal cells. The pelleted stromal cells are finally incubated with red blood cell lysis solution and centrifuged one more time. This enzymatic procedure generates tissue fragments that should be removed before cell plating through a $100-150 \mu \mathrm{m}$ nylon mesh $[3,4,5]$.

Irrespective of the source of tissue, enzymatic digestion is time consuming and expensive, especially when applied to large volumes of tissue decreased cell viability due to lytic activity is also a problem with this method [6,7].

\section{Mechanical separation:}

A novel method of isolating MSC from lipoaspirate samples based on mechanical tissue dissociation. Despite the major differences between the enzymatic and mechanic methods, similar populations of MSC have been isolated [6].

The population of cells derived from mechanical process was positive for mesenchymal surface markers such as CD90 and CD105. They were also positive for $\mathrm{CD} 34$, which is reported only in adipose tissue-derived mesenchymal cells [8].
Mechanical dissociation consists basically of two steps: Dissociation of adipose tissue concomitantly with red blood cell lysis, followed by centrifugation. There are no visible tissue fragments, and it is not necessary to filter the cell suspension. The ease of mechanical digestion reduces considerably both time and cost, and does not interfere with cell viability [6].

\section{Stem cell enriched fat transfer (Liposowing):}

Recently, medical techniques have been proposed for tissue regeneration using autologous adult fat stem cells obtained with liposuction to restore the regular volumes of adipose tissue in the body and especially in the face. The regeneration of the face adipose tissue follows the outline of a technique already in use: the lipofilling. This process is based on fat cells obtained with lowpressure suction and further centrifugation to separate fat cells from the stromal-vascular connective tissue. The aim is to infiltrate only intact cells, able to survive in their new home [9].

For the technique of regeneration basically normal fat cells and fat Stem cells are used, these are sown in small amounts to stimulate the formation of new adipose tissue (liposowing).

J. Victor Garcia and Maurizio Ceccarelli have developed a simple technique to enrich stem cells in the area of collection for Liposowing [10]. The explanation of why fat is so rich in adult stem cells can be sought in the biological function of this tissue: the energy storage. The adipocyte is able to significantly increase its volume to collect energy in the triglycerides form. But when its volume is very high (higher than $170 \%$ of normal volume) the adipocyte stimulates the formation of new adipose tissue by activating the differentiation of stem cells in the stroma-vascular connective tissue. The liposintetic stimulus leads to adipocyte hypertrophy that, at a certain volume, stimulates perivascular stem cell propagation and differentiation. The stimulus for preadipocytes mitosis and differentiation follows mainly the increase of the insulin rate for the receptor down regulation and the liberation IGF-1 in the hypertrophic adipocyte [11].

The liposowing differs therefore from lipofilling in that the donor area is enriched with stem cells prior to harvest while lipofilling stem cells is isolated and then the fat is enriched with them [10].

To improve the end results; harvest and reinjection techniques have been recently refined. In addition the concomitant use of autologous stem cells and the stromal vascular fraction (SVF) has been proposed as an adjunct to improve the amount 
of retained fat. In this study we will utilize both the mechanical and enzymatic separation of the SVF with recent refinement of both techniques in order to compare the clinical outcome of each method.

The aim of this study is to evaluate the effect of two types of isolation of lipograft in the face and their persistence and the changes they will produce.

\section{MATERIAL AND METHODS}

This study was conducted on 50 patients complaining of facial ageing and facial scars whether post traumatic or post burn from the period between January 2013 and December 2014.

The sample population was divided into two groups:

Group A: Underwent fat processing using the mechanical mesh/gauze to separate the SVF (39 patients) in order to obtain micro and nanofat grafts.

Group B: Underwent fat processing using the enzymatic/collagenase technique to separate the SVF (11 patients).

\section{Inclusion criteria:}

- Patients with signs of facial ageing.

- Patients with facial defects and disharmonies.

- Patients with post traumatic facial scars.

- Patients with postburn scarring of the face.

\section{Exclusion criteria:}

- Chronic heavy smokers.

- Irregular eating habits.

- Patients on a diet program to lose weight.

- Medical Conditions including severe hypertension, renal disease, steroid dependent asthma, immuno-suppressed disease, Systemic Lupus, uncontrolled diabetes.

Patient evaluation and Pre-operative preparation: Medical history:

History taking needs to consider psychological, oncological, and general medical aspects the history included the following points; age, family history, prior breast disease.

The physical examination included examining the facial features of the patient while standing, while laughing and while in resting position. Both frontal and profile views were observed. Digital photography using a standardized 3D camera was done in 6 different views. Accurate markings of the fine wrinkles and sagging of the tissues was done using a single use marking pen to avoid infection.

\section{Intra-operative:}

Anesthesia:

The procedure was performed under general anesthesia or local anesthesia.

\section{Antibiotics:}

Patients were given $2 \mathrm{gms}$ of antibiotic IV intraoperatively, 6 hours post operatively and then for the next 4 days in an intramuscular form.

\section{Sterilization:}

Alcohol or Betadine for sterilization of harvesting sites injection sites, reaching up to the neck.

\section{Draping:}

Covering the neck, arms, forearms, hands, legs and feet.

\section{Incision:}

Small skin incision using a number 11 blade was done to allow for infiltration of the tumescent solution in donor areas. The solution injected was prepared as follows.

To each $1000 \mathrm{ml}$ of $9 \%$ saline solution warmed up to add the following:

- 1 ampule of adrenaline $1 \mathrm{mg} / \mathrm{ml}$.

- $35 \mathrm{cc}$ lidocaine $2 \%$ if the procedure is done with local anesthesia.

- $10 \mathrm{ml}$ sodium bicarbonate if the procedure is done with local anesthesia.

\section{Surgical technique: Method of Aspiration:}

The suction cannula had a diameter of 2 or $3 \mathrm{~mm}$, with multiple sharp 1-mm holes at its top segment. The limited diameter of the cannula holes is important for obtaining small particles to be injected through a 23-gauge needle. We used harvesting cannulas of 2 to $3 \mathrm{~mm}$ in diameter, which contain multiple small-diameter (1-mm) sharp holes. A yield of $1 \mathrm{~mL}$ of fat per $5 \mathrm{~mL}$ of aspirate can be expected, which is relatively low compared with that obtained by classical harvesting cannulas and is achievable because of the multiple, fine, sharp holes of the cannula.

The key to successful fat grafting is the use of micro-grafts [12]. The radius of the fat particle is inversely proportional to its contact surface. This means that for the same volume of injected fat, reducing. 


\section{Group A:}

A 2-mm stab incision was made along the skintension lines with a number 11 blade. Liposuction was performed manually, with sterile syringes or with a suction device and a sterile canister. If the desired volume of fat was less than $10 \mathrm{~mL}$, the fat was harvested with a cannula on a $10 \mathrm{~mL}$ Luer-Lok syringe. Volumes of more than $10 \mathrm{~mL}$ were harvested with a suction device and a sterile canister.

The suction cannula had a diameter of 2 or $3 \mathrm{~mm}$, with multiple sharp 1-mm holes at its top segment. The limited diameter of the cannula holes is important for obtaining small particles to be injected through a 23-gauge needle.

The sharp edges of the holes augment the harvesting yield of the suctioned fat, because the cannula works like a rasp in the donor area. A yield of $1 \mathrm{~mL}$ of fat graft per $5 \mathrm{~mL}$ of aspirate can be expected.

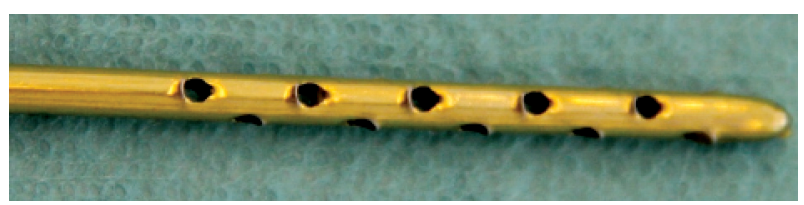

Fig. (1): The harvesting cannula consists of a multiport sharp cannula, $2 \mathrm{~mm}$ in diameter, with holes of $1 \mathrm{~mm}$ in diameter. Note the sharp edges of the holes, which transform the cannula into a rasp, augmenting the harvesting yield of this small-holed cannula.

A sterile nylon cloth with $0.5-\mathrm{mm}$ mesh size was mounted over a sterile canister, and the supernatant was poured over it. The fat was rinsed with normal saline and transferred into $10 \mathrm{cc}$ syringes. With the help of a double Luer-Lok adapter, the fat was brought into $1 \mathrm{cc}$ syringes. An $0.7 \mathrm{~mm}$ blunt cannula was adjusted over the $1 \mathrm{cc}$ syringe and was used for injection of the deep folds. A 23-gauge needle was mounted on the syringe for intradermal injection.

The injection was made in a superficial dermal plane. The skin was pinched, with the wrinkle lying between the thumb and index finger. Linear thread injection of the fat was performed while the needle was withdrawn.

Immediately after fat injection, the skin is blanched over the injected wrinkle. A slight overcorrection is advised because the blanching and overcorrection will normalize within a few hours, after resorption of the interstitial fluid. For deep wrinkles and folds separating the wrinkle from its deep attachments, subcision may be needed to achieve an optimal result. This can be achieved with a 17-gauge needle used as a "knife" under the skin. The 17-gauge needle is used only for subcision-not for intradermal injection. Intradermal fat injection can be performed as a supplementary and final procedure after classic lipofilling of deep folds (e.g., nasolabial folds), with fine, blunt-tip cannulas. This technique was utilized in most of the aging correction group and in some of the post traumatic, congenital asymmetry and irregularities of the face.

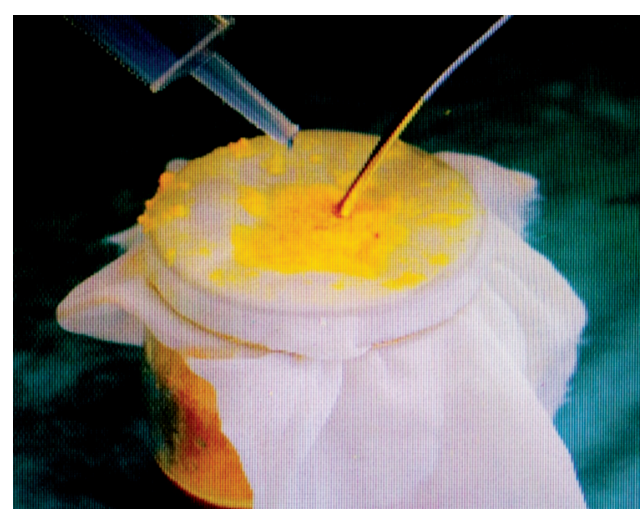

Fig. (2): A sterile canister with a $0.5-\mathrm{mm}$ mesh cloth is used to rinse the fat with a sterile normal saline solution.

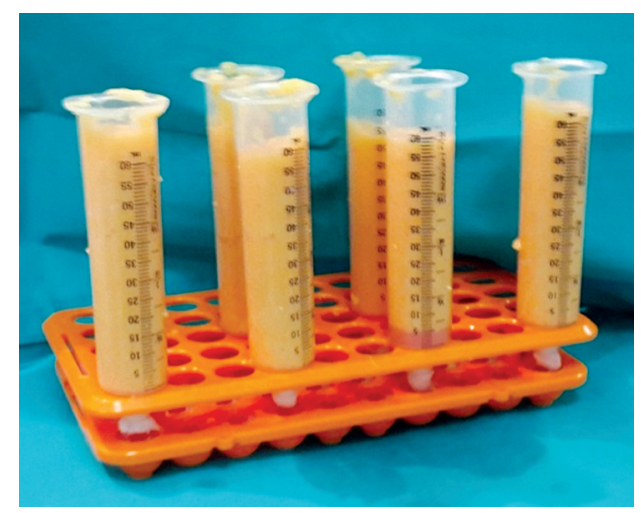

Fig. (3): Showing the fat transferred to sterile syringes.
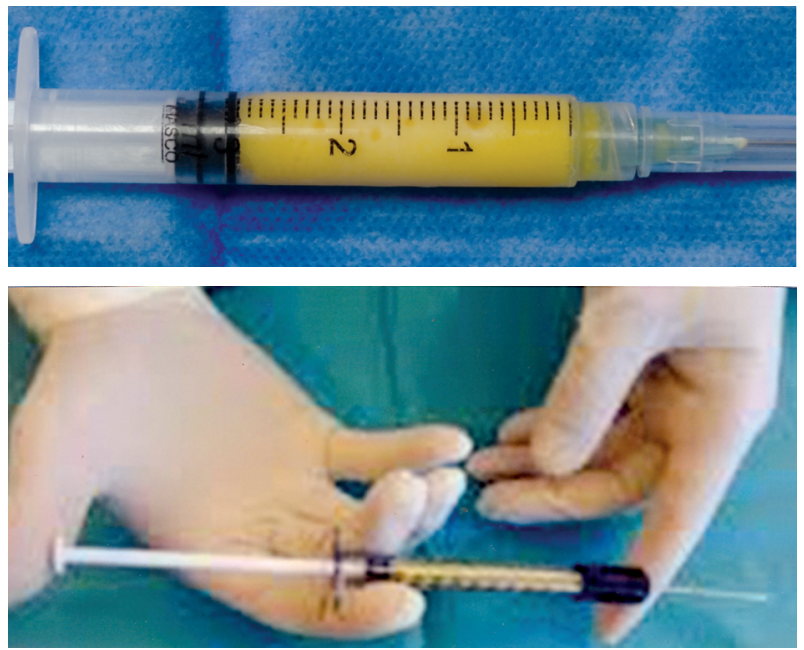

Fig. (4): Fat is then transferred to $1 \mathrm{cc}$ or $3 \mathrm{cc}$ sterile syringes using a female to female leur lock connector. 
A refinement of the mechanical processing technique was introduced by using the so-called Nanofat graft. The use of nanofat grafts was confined to managing postburn, posttraumatic scars, black halos and crows feet around the eye and perioral smoker's wrinkles.

The lipoaspirate was mechanically emulsified after rinsing. Emulsification of the fat was achieved by shifting the fat between two 10-cc syringes connected to each other by a female-to-female Luer-Lok connector. After 30 passes, the fat changed into an emulsion. At the end of the fragmentation process, the fat became liquid and took on a whitish appearance. After this emulsification process, the fatty liquid was again filtered over the sterile nylon cloth and the effluent was collected in a sterile recipient. This was done to remove the connective tissue remnants that would block the fine needles. This effluent is called "Nanofat". In many cases both microfat and nanofat grafts were combined to achieve satisfactory results.

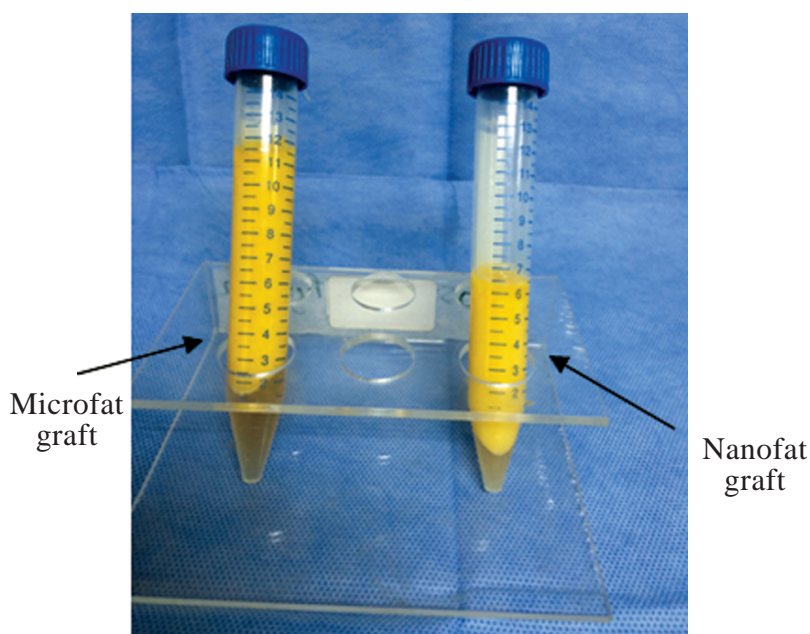

Fig. (5): Mechanical emulsification of microfat graft to yield nanofat graft.

\section{Group B: Stem Cell Isolation:}

Isolation of the adipose-derived stem cells from the different lipoaspirates Preparation of the SVF using enzymatic digestion required the following steps:

1- Lipoaspirate was washed with equal volume of Phosphate buffer solution (PBS) and left for 2-5 minutes. Decant the fluid portion that settled at the bottom of the tube.

2- Repeat the previous step for 3 times.

3- Wash the lipoaspirate again and add equal volume of collagenase $0.1 \%(0.3 \mathrm{u} / \mathrm{L})$.

4- Mixture was incubated in a culture flask in a gyratory shaker bath at $37 \mathrm{C}$ for 1 hour (500 shakes for 1 hour).

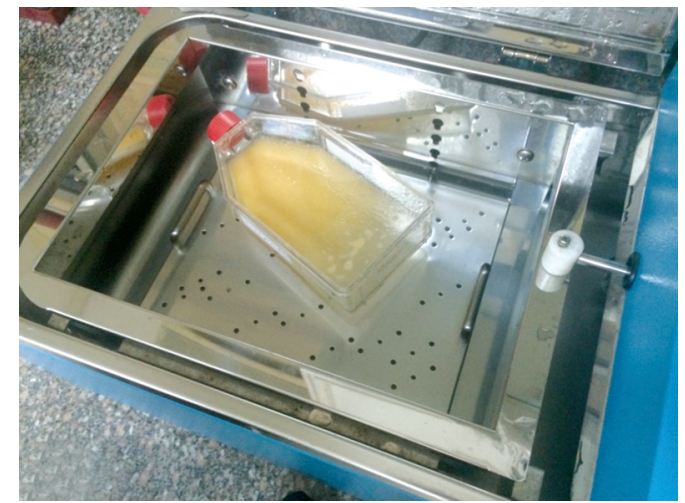

Fig. (6): Mixture incubated in a culture flask in a gyratory shaker bath.

5- Pour in a Falcon Tube and add equal volume of DMEM (Dulbeccos Modified Eagle Medium).

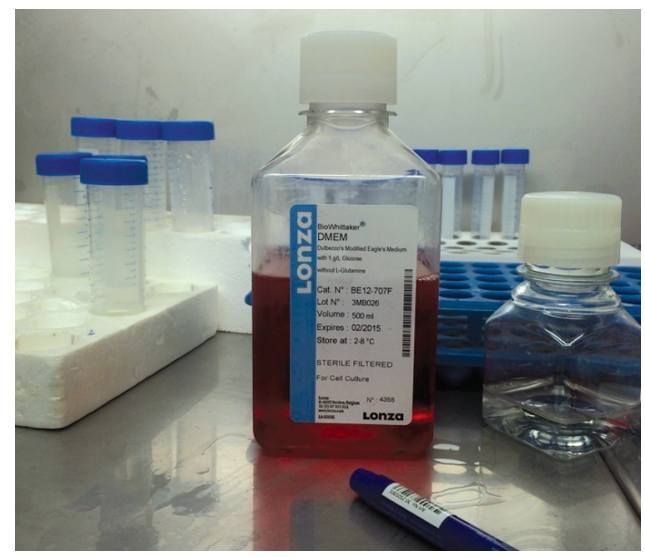

Fig. (7): Showing DMEM (Dulbeccos Modified Eagle Medium).

6- Centrifugation for 10min@ 4000rpm. Mixture separates into upper fatty layer, buffer and cell pellet. The overlying fluid and adipose phases were aspirated and discarded.

7- The stromal cell pellet was resuspended in phosphate-buffered saline $(5 \mathrm{ml} / 10 \mathrm{ml})$. The mixture was now ready for injection either separately or was mixed with PRP or lipoasprated fat.

\section{Post-operative:}

Patients were allowed to go home on the same day. Cold compresses and a local antibiotic cream was advised to the patient every 6 hours.

\section{Follow-up:}

Follow-up at 1 week,, 1 month, 6 months, 1 year.

All the 50 patients have been followed and evaluated clinically and using $3 D$ digital camera. Assessment was done according to the following:

- Improvement in the quality of the skin.

- Improvement of facial disharmonies. 
- Improvement in the colour of the skin.

- Improvement of the scar size and shape.

Statistical methodology:

Analysis of data was done by SPSS software (statistical program for social science version 12) as follows:

- Description of quantitative variables as mean, SD and range.

- Paired sample $t$-test test was used to compare qualitative variables between groups.

- Fisher exact test was used instead of chi-square when one expected cell $<5$.

p-value:

- $p$-value $>0.05$ insignificant.

$\bullet p<0.05$ significant.

$\cdot p<0.01$ highly significant.

\section{RESULTS}

This table demonstrates the demographic data of both groups of our study which includes 50 patients divided into group (A) and group (B). In group A total number of patients was 39 patients with 5 males and 34 females were operated upon. In group B all 11 females were operated upon. The age mean of Group (A) was $44.18 \pm 12.25$ while in group (B) was $48.73 \pm 14.89$.

Table (1): Comparison between two studied groups according to demographic data.

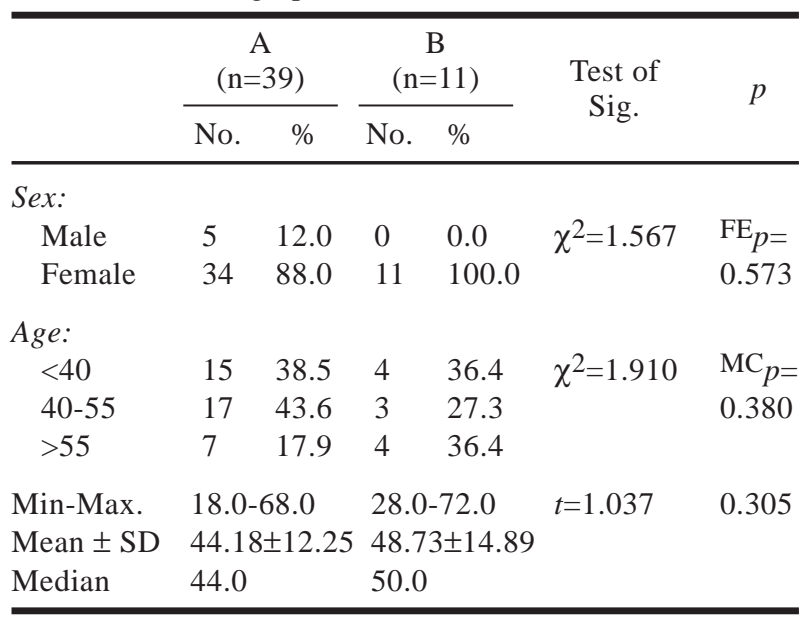

The mean amount of fat aspirated from group A was $342.31 \mathrm{cc} \pm 102.64$ while in group B was $345.5 \pm 41.56 \mathrm{cc}$.

The mean amount of fat injected in Group A patients was $37.97 \pm 17.74$ while in group B $34.91 \pm$ 6.64 .
Table (2): Comparison between two studied groups according to amount of fat aspirated and injected.

\begin{tabular}{|c|c|c|c|c|}
\hline & $\underset{(n=39)}{A}$ & $\begin{array}{c}\mathrm{B} \\
(\mathrm{n}=11)\end{array}$ & $t$ & $p$ \\
\hline \multicolumn{5}{|l|}{$\begin{array}{l}\text { Amount of fat } \\
\text { aspirated: }\end{array}$} \\
\hline Min-Max. & $150.0-600.0$ & $300.0-400.0$ & & \\
\hline Mean \pm SD & $342.31 \pm 102.94$ & $345.45 \pm 41.56$ & 0.152 & 0.88 \\
\hline Median & 300.0 & 350.0 & & \\
\hline \multicolumn{5}{|l|}{$\begin{array}{l}\text { Amount of fat } \\
\text { injected: }\end{array}$} \\
\hline injected: & & & & \\
\hline Min-Max. & $8.0-70.0$ & $25.0-45.0$ & & \\
\hline Mean \pm SD & $37.97 \pm 17.74$ & $34.91 \pm 6.64$ & 0.882 & 0.38 \\
\hline Median & 38.0 & 36.0 & & \\
\hline
\end{tabular}

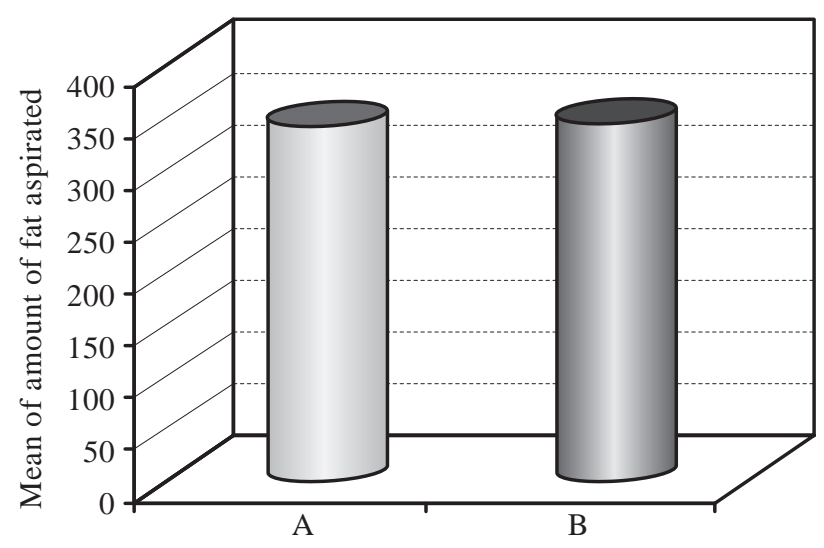

Fig. (8): Comparison between two studied groups according to amount of fat aspirated.

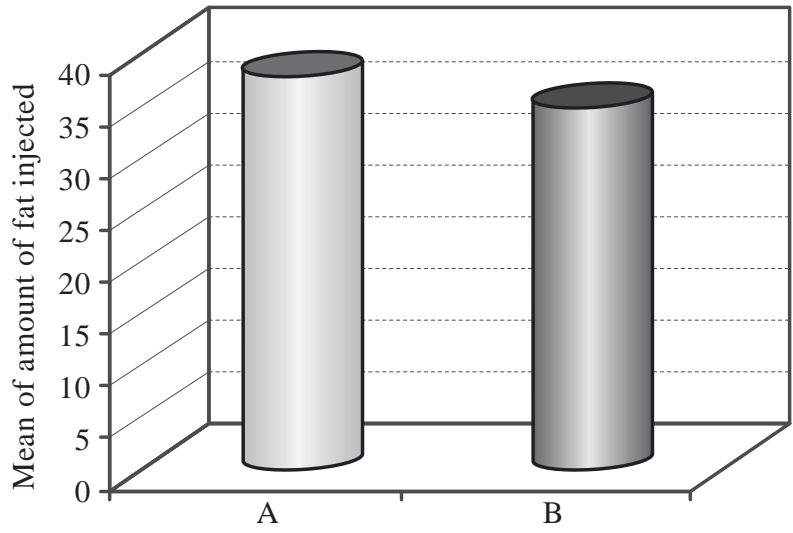

Fig. (9): Comparison between two studied groups according to amount of fat injected.

In group A 30 patients seeked facial rejuvenation representing $76.9 \%$ while black halos represented 4 patients $10.3 \%$ Another 4 post traumatic cases $10.3 \%$. One postburn case representing $2.6 \%$ was operated upon. In group B 7 patients seeked rejuvenation representing $70 \%$ while in $30 \%$ cases where post traumatic. 
Table (3): Comparison between two studied groups according to second touch up and PT/PB/ Rejuvenatation.

\begin{tabular}{|c|c|c|c|c|c|c|}
\hline & \multicolumn{2}{|c|}{$\begin{array}{c}\mathrm{A} \\
(\mathrm{n}=39)\end{array}$} & \multicolumn{2}{|c|}{$\begin{array}{c}\mathrm{B} \\
(\mathrm{n}=11)\end{array}$} & \multirow[t]{2}{*}{$\chi^{2}$} & \multirow[t]{2}{*}{$p$} \\
\hline & No. & $\%$ & No. & $\%$ & & \\
\hline \multicolumn{7}{|l|}{ Second touch up: } \\
\hline Negative & 33 & 84.6 & 11 & 100.0 & 1.923 & $\mathrm{FE}_{p=}$ \\
\hline Positive & 6 & 15.4 & 0 & 0.0 & & 0.317 \\
\hline \multicolumn{7}{|l|}{$P T / P B /$} \\
\hline \multicolumn{7}{|l|}{ Rejuvenatation: } \\
\hline Black halos & 4 & 10.3 & 0 & 0.0 & 2.140 & $\mathrm{MC}_{p=}$ \\
\hline Rejuvenatation & 30 & 76.9 & 7 & 70.0 & & 0.605 \\
\hline Post burn & 1 & 2.6 & 0 & 0.0 & & \\
\hline Post traumatic & 4 & 10.3 & 3 & 30.0 & & \\
\hline
\end{tabular}

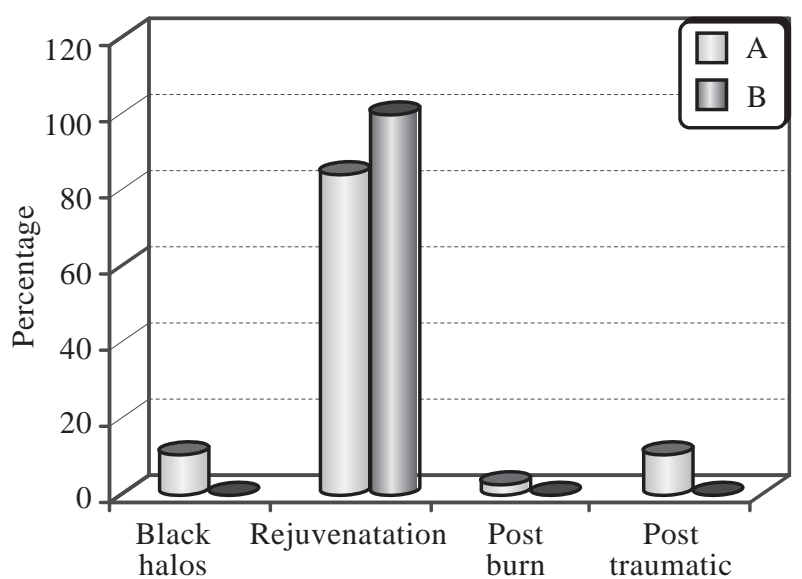

$\mathrm{PT} / \mathrm{TB} /$ rejuvenatation

Fig. (10): Comparison between two studied groups according to second touch up and PT/PB/ Rejuvenatation.

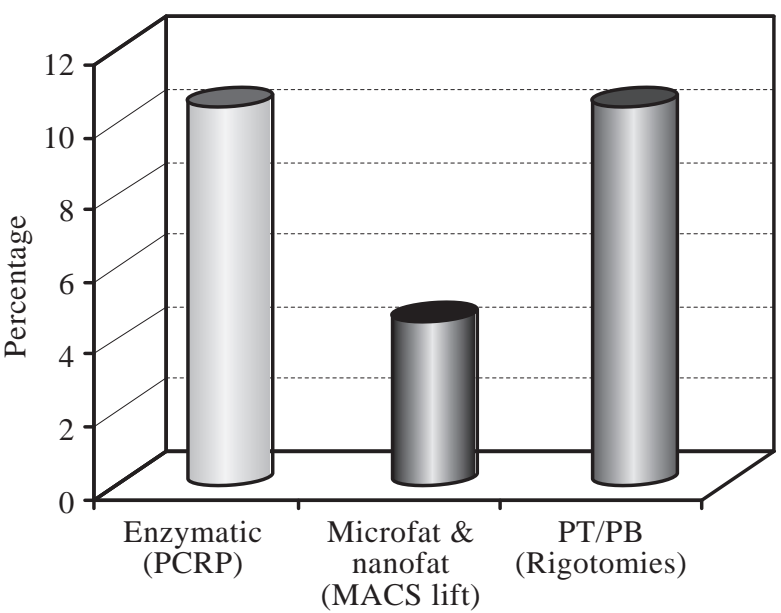

Additional technique

Fig. (11): Distribution of the studied cases according to additional technique.

Table (4): Statistical analysis of the studied cases according to Amount of fat aspirated and injected $(n=50)$.

\begin{tabular}{llll}
\hline & Min-Max. & Mean \pm SD & Median \\
\hline Amount of fat aspirated & $150.0-600.0$ & $343.0 \pm 92.59$ & 300.0 \\
Amount of fat injected & $8.0-70.0$ & $37.30 \pm 15.96$ & 38.0 \\
\hline
\end{tabular}

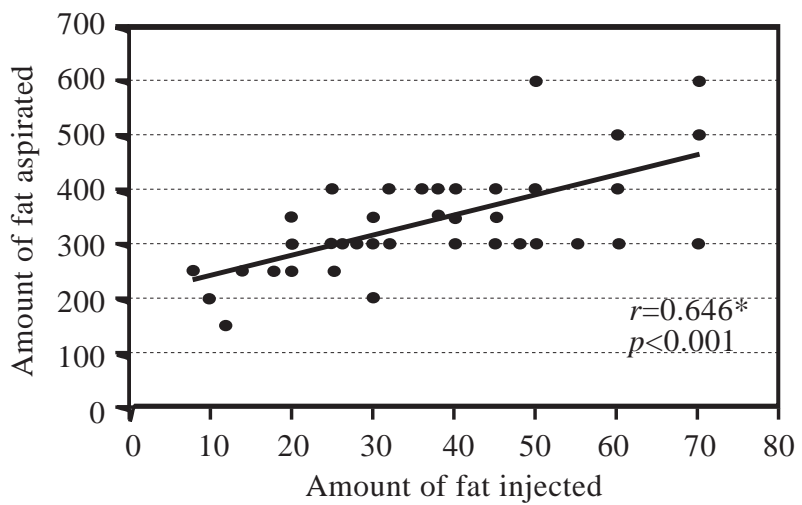

Fig. (12): Correlation between amounts of fat injected with amount of fat aspirated.

The amount of fat injected increases with age which represents a direct proportionality between the age and amount of fat injected. The same theory lies with the amount aspirated which also increases in older age groups.

Table (5): Correlation between age with Amount of fat aspirated and injected $(n=50)$.

\begin{tabular}{lcc}
\hline & \multicolumn{2}{c}{ Amount of fat injected } \\
\cline { 2 - 3 } & $r$ & $p$ \\
\hline Age & $0.449^{*}$ & 0.002 \\
Amount of fat aspirated & $0.646^{*}$ & $<0.001$ \\
\hline
\end{tabular}

$r$ : Pearson coefficient. $\quad *$ : Statistically significant at $p \leq 0.05$.

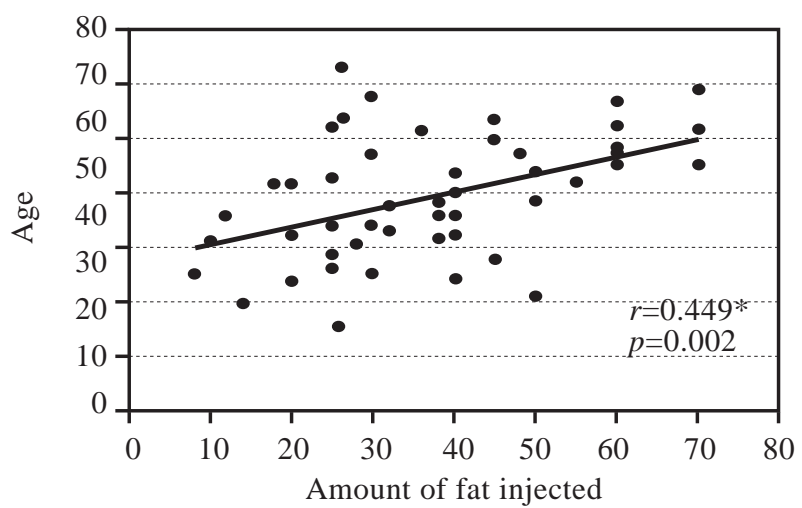

Fig. (13): Correlation between age with amount of fat injected.

\section{Complications:}

Our most common complication was bruising, redness and swelling in only 5 cases representing $10 \%$. Resorption was our second most common complication in only 4 cases representing $8 \%$.

Table (6): Distribution of the studied cases according to complications.

\begin{tabular}{lll}
\hline & No. & $\%$ \\
\hline Complications: & & \\
Bruising, Redness, Swelling & 5 & 10.0 \\
Infection & 0 & 0.0 \\
Resorption & 4 & 8.0 \\
Fat nodules/Cyst/granulomas & 0 & 0.0 \\
Fat necrosis & 0 & 0.0 \\
\hline
\end{tabular}




\section{Clinical Cases}

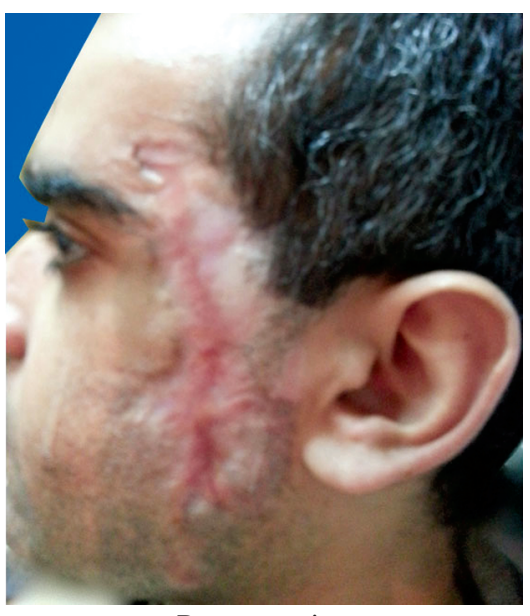

Pre operative

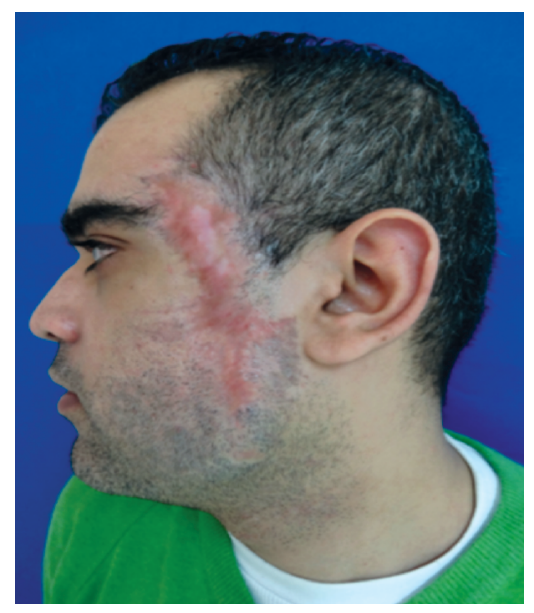

Immediate post operative

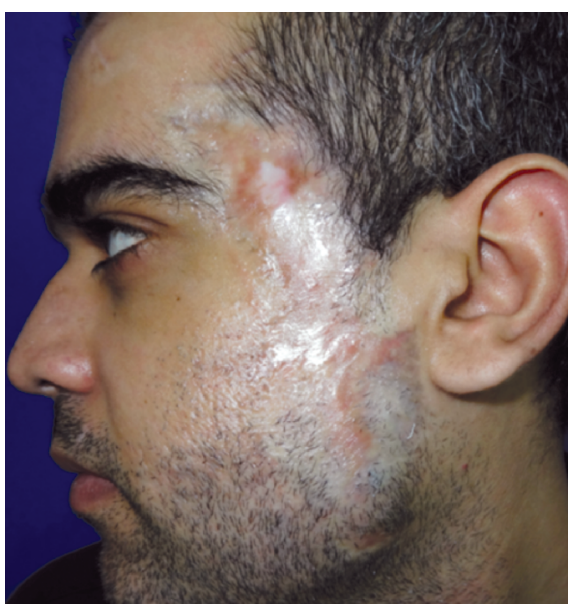

Six months post operative

Case (1):

- Mr. Ahmed, a 25 year old male patient, complaining of multiple post traumatic scar in the temporal region up to the angle of mandible as a result of an RTA of the face.

- Revision of the scar was done in one session.

- $250 \mathrm{cc}$ of fat was aspirated from the lower abdomen

- 100 cc yield of MFG, after mechanically washing the fat with normal cold saline, was injected intralesionally and subdermal.

- Patient was also treated with multiple PRP sessions.

\section{Histopathological assessment:}

After one year of fat grafting, excision and revision of the scar was done, two specimens were sent for assessment. The histopathological assessment demonstrates serial sections showing normal epidermis with absent melanin pigments. The upper dermis shows focal infiltration by chronic mononuclear inflammatory cells including frequent melanin laden histiocytes and macrophages. Underneath subcutaneous fat is hypertrophied manifested by increase in size of individual lipocytes. The fatty tissue is including quite frequent scattered capillaries with mild perivascular infiltration by chronic mononuclear inflammatory cells mainly lymphocytes and plasma cells among hemosiderin laden histiocytes. No lipogranulomatous fat necrosis is present.

\section{Clinical outcome and observation:}

The width and breadth of the scar have both decreased in size by $50 \%$. The colour of the skin has changed showing areas of hypopigmentation and decrease in areas of hyperemia. The texture of the skin has improved with no more dryness of the skin. Change in skin pliability and softness.

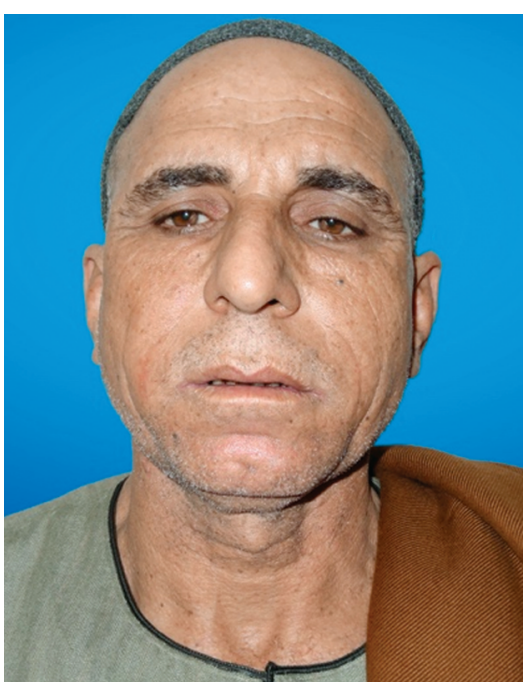

Pre operative

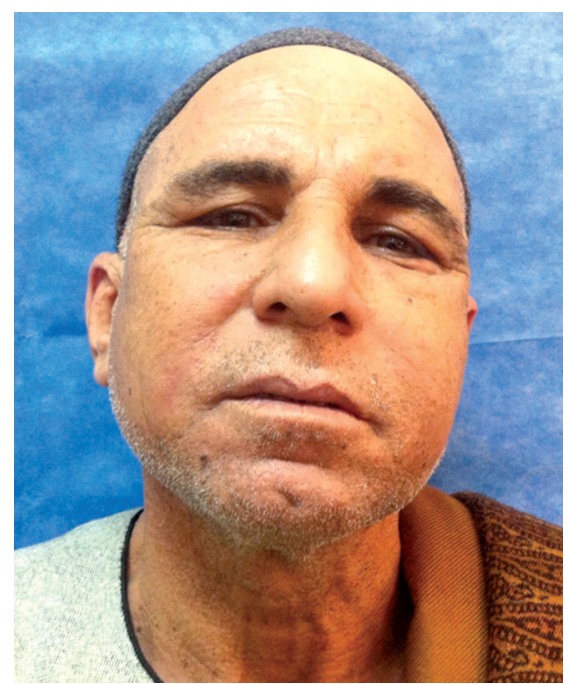

Six months post operative

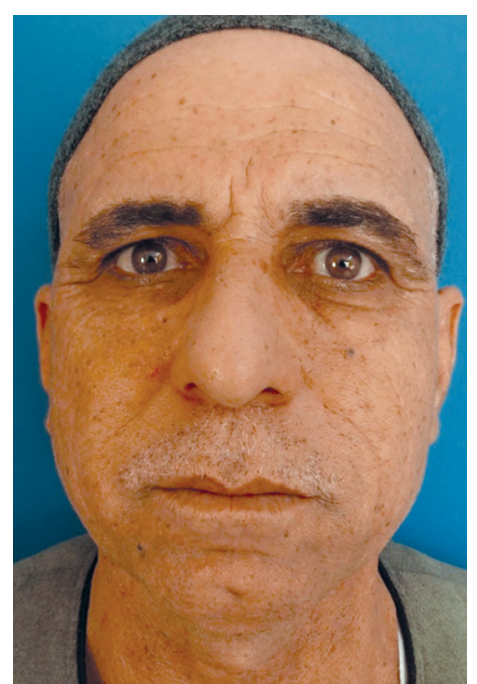

One year post operative

Case (2):

- A 67 year old male patient, complaining of facial aging and fine wrinkles in the forehead.

- 600cc of fat was aspirated from the lower abdomen.

- 200cc yield of lipograft in the form of 150cc MFG and 25cc NFG.

- These lipografts were injected in the forehead, Nasolabial folds, Marionette groove, hollow temples, and to eliminate crow's feet. 


\section{Clinical outcome and observation:}

Disappearance of the skin ageing signs. Disappearance of the brown spots and keratotic lesions. Change in the colour and texture of the skin together with improved dryness of the skin. Filling of the Nasolabial folds, marionette groove and forehead wrinkles. An automatic temporal lift as a result of MFG that was injected.

Case (3):

- A 22 year old female patient, complaining of unilateral left hemifacial microsomia.

- 200cc of fat was aspirated from the inner thighs to yield.

- $100 \mathrm{cc}$ of MFG.

- MF was injected in different planes to correct microsomia.

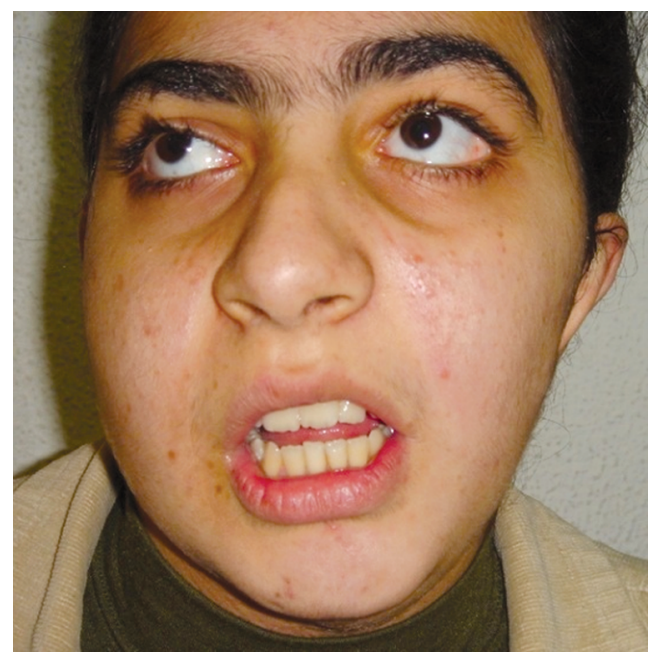

Pre operative

\section{Clinical outcome and observation:}

Filling of the left side of the face has shown over correction and hypertrophy of the left side more than the normal side after one year of injection. Therefore the patient asked for MFG of the normal side.

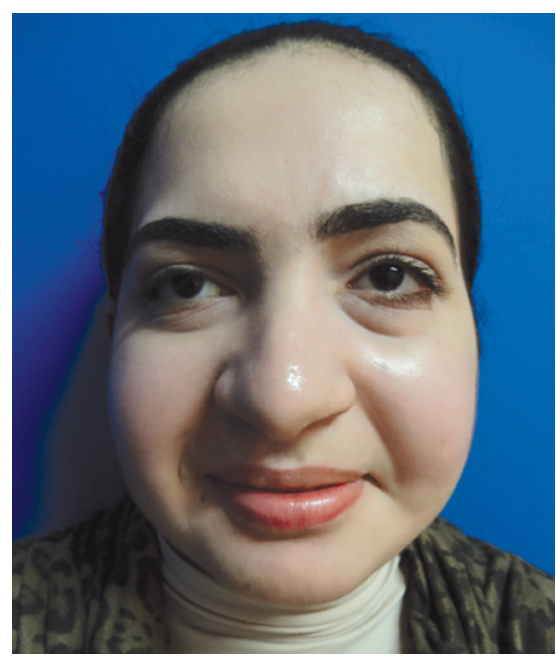

One year post operative

\section{Clinical outcome and observation:}

Filling of the left side of the face has shown over correction and hypertrophy of the left side more than the normal side after one year of injection. Therefore the patient asked for MFG of the normal side.

Case (4):

-A 24-year-old female patient, complaining of post-bariatric facial atrophy.

- 500cc of fat was aspirated from the lower abdomen, suprapubic and thoracic back to correct facial sagging.

- $100 \mathrm{cc}$ of MFG was injected in different planes to fill the face

- Patient needed a second session in which $150 \mathrm{cc}$ of fat was aspirated from the lower abdomen to yield 30cc of MFG and $10 \mathrm{cc}$ of NFG.

- NFG was used to correct black halos.

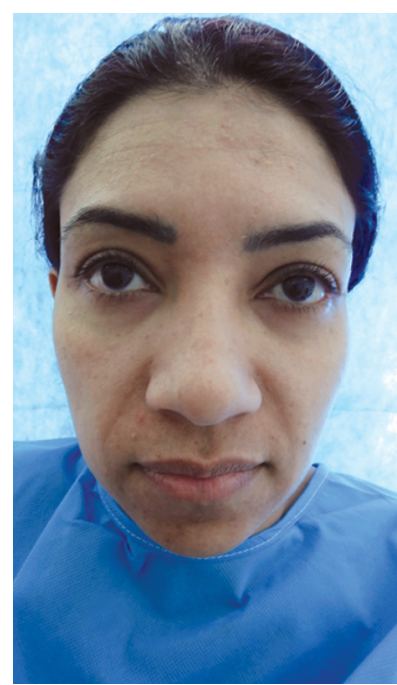

Pre operative

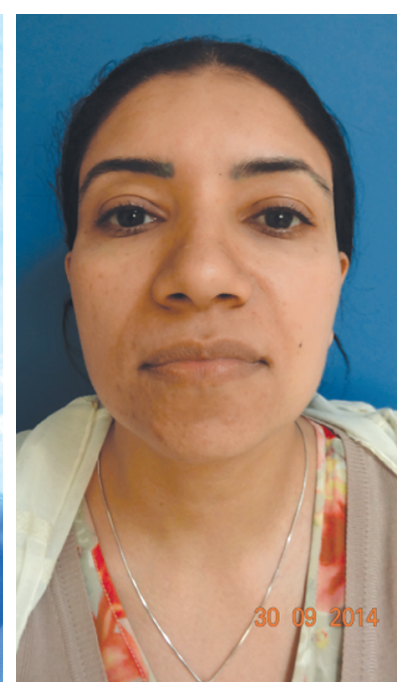

Pre operative

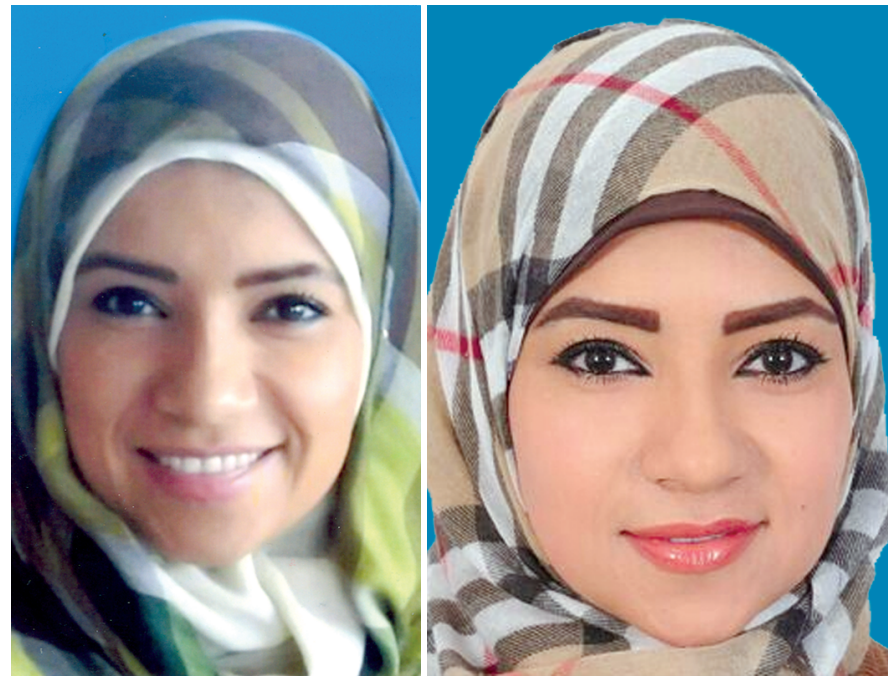

6 months
Post operative 


\section{Clinical outcome and observation:}

Sagging of the face has disappeared with correction of the grooves that were filled. The skin texture improved together with skin pliability. Decrease and disappearance of the post acne scars together with decreased dryness of the skin. 6 months postoperative black halos started to decrease gradually.

\section{Case (5):}

Case of a posttraumatic scar in the left side of the face. Patient was treated with lipografting using the enzymatic technique. 300CC was aspirated from the lower abdomen. Injection was done in different planes. Multiple rigottomies were also done.

\section{Clinical outcome and observation:}

Complete Improvement of the skin texture, colour and pliability. Disappearnce of the scars from the face. No more hyper pigmentation around the scar or postacne scars. No more hyperemia around the scar. No more dryness or thick texture of the skin.

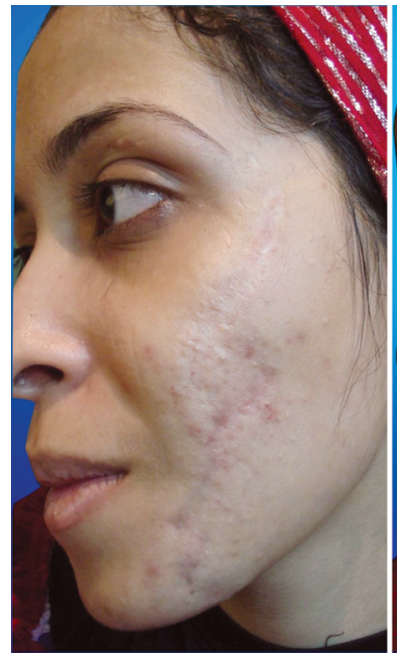

Pre operative

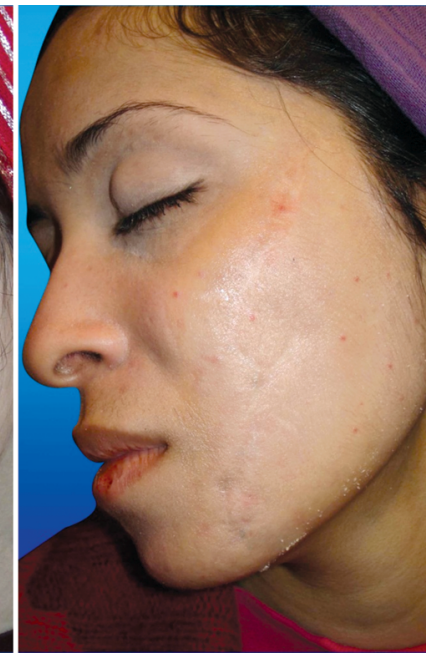

Post operative

\section{DISCUSSION}

Today, minimally invasive surgical techniques are welcomed in many surgical disciplines as well as in aesthetic surgery techniques, as long as the quality of the results remain as good with minimal complication rate, little or no hospital stay, a swift recovery and with no or inconspicuous scars. These criteria are fulfilled by the different techniques of fat grafting and preparation of the Stromal Vascular Fraction.

During this study, it was noted that results improved as physicians gained experience with the technique. As with any other technical skill, proficiency at producing excellent results is conditional on practice and perfection of the technique.

Several factors have an effect on autologous graft survival rates including the method of fat harvesting, the volume of graft injected into a single area, the quality of the harvested fat, and the selection of cannulas for aspiration and injection.

Kishi et al., performed the same study but on 18 cadavers and proved that the thoracic back is the site with the highest concentration of stromal vascular cell concentration [13]. The data proved that, in young female patients, the lower abdomen region has a higher concentration of stromal vascular cells than the flank but that no difference is found when comparing it with the inner thigh.

In our study, although group (B) confined with the previous study in showing similar adipocyte count yet group (A) with variable donor sites from lower abdomen, thighs and thoracic back show no significant difference.

Many suggest that adipose-derived stem cells play an important role in fat grafting [2]. The literature shows high variability in stromal vascular fraction counts, with a small percentage being true adipose-derived stem cells. For example, the restore-2 trial reports 295,000 cells in the stromal vascular fraction of $1 \mathrm{~g}$ of fat, [14] whereas Yoshimura et al., reported 1,310,000 cells [2]. In this study, we also found large variability in the total number of stromal vascular fraction cells as demonstrated in the SVC count in (Table 10), we found that the stromal vascular fraction in the mechanical technique group (A) contained a lower cell count with a higher proportion of adipose-derived stem cells. We believe this is because mechanical technique group (A) is more effective at removal of blood cells, which is the major cellular component of the stromal vascular fraction. We consistently saw an inversely proportional relationship between the total number of stromal vascular fraction cells and the percentage of adipose-derived stem cells, suggesting that washing undigested fat effectively removes blood without washing away adiposederived stem cells.

Al Harbi et al., demonstrated a slight but not a significant differences in the yields of ADSC by either techniques the mechanical and the enzymatic [15]. They claimed that the enzymatic technique can increase the number of ADSC by $22.4 \%$. The clinical implications of this technique superiority was observed in decreasing the amount of volume loss and the rapid effect on the skin dermal thickness, changes in pliability, colour and appearance. 
The need for secondary retouches in group (B) was observed to be less except after adding the nanofat graft technique to group (A) where we believe that the number of ADSC's was increased and clinical effects were more profund. The role of adipose-derived stem cells in fat grafting remains unclear. Mechanistically, they seem to contribute to neovascularization of the graft by means of angiogenic signals and endothelial cell differentiation [16]. Adipose-derived stem cells represent a very small fraction of the tissue grafted, and the numbers of ADSC obtained by both techniques was found to be equivalent.

Both methods in both groups resulted in equivalent weight and histology but many believed that a closed technique as in group (B) is superior, postulating that exposure to air harms fat and risks contamination [17]. However, our data and others' data do not support these claims.

The impact of the technique on the number of ADSC's and the viability of the prepared lipograft aspirate is the main factor in determining the outcome of each technique. This was similar to the study of Al Harbi et al., 2013, [15].

We have observed the clinical results of both group (A) and group (B) with almost similar improvement effects in rejuvenation of facial aging effects and the effect on facial scars according to Vancover scale. Improvements in clinical criteria of skin pliability, scar thickness, colour, itching were observed to improve with no significant difference between both groups.

\section{Conclusion:}

The techniques of fat processing both the mechanical and enzymatic techniques have demonstrated valuable clinical outcomes in all indications. Although the enzymatic technique demonstrated valuable results in concentrating the ADSC's with less need for secondary retouches. We observed an improvement in the mechanical group by adding the new technique of nanofat grafting which concentrates more ADSC'S in its component. More research is needed to purify the isolation of ADSC's and its growth and tissue stimulating factors are ready to be examined in future research.

\section{REFERENCES}

1- Fraser J.K., Zhu M., Wulur I. and Alfonso Z.: Adiposederived stem cells. Methods. Mol. Biol., 449: 59-67, 2008.

2- Yoshimura K., Sato K., Aoi N., Kurita M., Hirohi T. and Harii K.: Cell-assisted lipotransfer for cosmetic breast augmentation: Supportive use of adipose-derived stem/stromal cells. Aesthetic Plast Surg, 32 (1): 48-57, 2008.
3- Gimble J.M., Katz A.J. and Bunnell B.A.: Adipose-derived stem cells for regenerative medicine. Circ. Res., 100 (9): 1249-60, 2007.

4- Jing W.1., Lin Y., Wu L., Li X., Nie X., Liu L., et al.: Ectopic adipogenesis of preconditioned adipose-derived stromal cells in an alginate system. Cell and Tissue. Cell. Tissue. Res, 330 (3): 567-72, 2007.

5- Bunnell B.A., Flaat M., Gagliardi C., Patel B. and Ripoll C.: Adipose-derived stem cells: Isolation, expansion and differentiation. Methods., 45 (2): 115-20, 2008.

6- Baptista L.S., do Amaral R.J., Carias R.B., Aniceto M. and Claudio-da-Silva C.: BorojevicR. An alternative method for the isolation of mesenchymal stromal cells derived from lipoaspirate samples. Cytotherapy, 11 (6): 706-15, 2009.

7- Ishige I., Nagamura-Inoue T., Honda M.J., Harnprasopwat R., Kido M., Sugimoto M., et al.: Comparison of mesenchymal stem cells derived from arterial, venous, and Wharton's jelly explants of human umbilical cord. Int. J. Hematol., 90 (2): 261-9, 2009.

8- Planat-Benard V., Silvestre J.S., Cousin B., Andre M., Nibbelink M., Tamarat R., et al.: Plasticity of human adipose lineage cells toward endothelial cells: Physiological and therapeutic perspectives. Circulation, 109 (5): 656-63, 2004.

9- Ceccarelli M. and García J.V.: The Medical Face Lift: Face Tissue Regeneration, The Medical Letter Physiological, I (1), 2010.

10- Bunnell B.A., Flaat M., Gagliardi C., Patel B. and Ripoll C.: Adipose-derived stem cells: Isolation, expansion and differentiation. Methods, 45 (2): 115-20, 2008.

11- Avram M.M., Avram A.S. and James W.D.: Subcutaneous fat in normal and diseased states 3. Adipogenesis: From stem cell to fat cell. J. Am. Acad. Dermatol., 56 (3): 47292, 2008.

12- Tonnard P., Verpaele A., Peeters G., Hamdi M., Cornelissen M. and Declercq H.: Nanofat grafting: Basic research and clinical applications. Plast. Reconstr. Surg., 132: 101726, 2013

13- Kishi K., Imanishi N., Ohara H., Ninomiya R., Okabe K., Hattori N., et al.: Distribution of adipose-derived stem cells in adipose tissues from human cadavers. J. Plast. Reconstr. Aesthet. Surg., 63 (10): 1717-22, 2010.

14- Pérez-Cano R., Vranckx J.J, Lasso J.M., Calabrese C., Merck B., Milstein A.M., et al.: Prospective trial of adipose-derived regenerative cell (ADRC)-enriched fat grafting for partial mastectomy defects: The RESTORE2 trial. Eur. J. Surg. Oncol., 38 (5): 382-9, 2012.

15- Ishige I., Nagamura-Inoue T., Honda M.J., Harnprasopwat R., Kido M., Sugimoto M., et al.: Comparison of mesenchymal stem cells derived from arterial, venous, and Wharton's jelly explants of human umbilical cord. Int. J. Hematol., 90 (2): 261-9, 2009.

16- Miranville A., Heeschen C., Sengenès C., Curat C.A., Busse R. and Bouloumié A.: Improvement of postnatal neovascularization by human adipose tissue-derived stem cells. Circulation, 110 (3): 349-55, 2004.

17- Sommer B. and Sattler G.: Current concepts of fat graft survival: Histology of aspirated adipose tissue and review of the literature. Dermatol. Surg., 26 (12): 1159-1166, 2000. 